\title{
Modeling of the natural and technogenic risks dynamics
}

\author{
V.A. Minaev, R.O. Stepanov, A.O. Fadeev \\ m1va@yandex.ru | fao1@mail.ru | stepanovr@,bmstu.ru \\ Bauman Moscow State Technical University
}

\begin{abstract}
A general view of the model of risk assessment in the natural-technogenic system (NTS), considering the effects of natural and technogenic factors, is considered. The general solution of the system of differential equations describing the model is found. Two examples of the application of the model for the case of functionally similar natural and technogenic impacts are analyzed: (i) linear effects resulting in catastrophic seismic events; (ii) parabolic impacts that lead to creep, karst-deformation, subsidence and landslide processes. In addition, two new models of the dynamics of risks arising in a TCP under the influence of dangerous natural and technogenic factors are described. The presented models differ from each other in the types of effects: in the first model, they consider jointly parabolic (reflecting threats, the intensity of which gradually decreases with distance from the epicenter) and linear types of effects (reflecting suddenly arising threats), in the second model, the analysis of such types of impacts as parabolic and hyperbolic (reflecting threats whose intensity decreases sharply over time) is carried out. It is concluded that, on the basis of the considered models, it is possible to accurately describe almost any type of combined natural and technological impact and also make a special "atlas" of complex effects on the NTS for preventive "playing" of various situations and developing effective counteraction to emerging dangers from the departments of the Ministry of Emergencies and other structures.
\end{abstract}

Keywords: modeling, differential equations, assessment, natural and technological risks, danger, counteraction.

\section{Introduction}

A complex combination of natural and anthropogenic factors that cause dangerous threats to the health and life of the population living in certain territories, as well as material objects, including critical ones located on them, determines the researches of natural and technogenic risks, which are devoted to a number of modern scientific papers [1-4], including scientific works on mathematical modeling of risks [5-7].

To ensure the safety of population and territories from the development of hazardous natural and technogenic processes in Russia, they are guided by the strategies indicated in the State Scientific and Technical Program «Safety of the population and national economic facilities, considering the risk of natural and technogenic disasters»» [8]:

1) prevention of the causes of natural and technogenic accidents and catastrophes and ensuring of facilities that are characterized by technogenic hazards;

2) prevention and localization of a dangerous situation that causes a chain reaction of events leading to a natural and technogenic accident or disaster;

3) maximum possible neutralization and rapid elimination of the effects of dangerous natural and technogenic factors on people and the environment.

It should be taken into account that extreme events, the implementation of which is unlikely from the point of view of statistics, reflect the "tail" values of the General population, as a rule, are underestimated by researchers. However, the consequences of such events are very large and dangerous (earthquakes, severe floods, super fires, mudslides, etc.) [9].

One of the most important methods for assessing natural and technogenic risks in natural-technical systems [NTS] is the method of mathematical modeling [10].

This article presents dynamic models of natural and technogenic risk in relation to systems exposed to complex external influences.

\section{Materials and method}

\section{Risk modeling for functionally similar natural and} technogenic influences

Assume risk is a two-dimensional vector function, where $r_{1}(t)$ - natural risk change function, $r_{2}(t)-$ technogenic risk change function. We represent the risk function in the form of a system of differential equations [11]:

$$
\left\{\begin{array}{l}
r^{\prime}{ }_{1}(t)=a_{1} \cdot r_{1}(t)+b_{1} \cdot r_{2}(t)+L_{1}(t), \\
r^{\prime}{ }_{2}(t)=a_{2} \cdot r_{1}(t)+b_{2} \cdot r_{2}(t)+L_{2}(t),
\end{array}\right.
$$

where $a_{1}, a_{2}, b_{1}, b_{2}-$ constant coefficients that reflect the response of the NTS to the effects of dangerous natural and technogenic factors; $L_{1}(t), L_{2}(t)$ - functions that describe the intensity of impacts on the NTS of external natural and technogenic factors, respectively.

The General solution of system (1) for zero functions $L_{1}(t), L_{2}(t)$ for the case of a positive discriminant of its characteristic equation is written as:

$$
\left\{\begin{array}{c}
r_{1}(t)=C_{1} \cdot e^{\lambda_{1} \cdot t}+C_{2} \cdot e^{\lambda_{2} \cdot t}, \\
r_{2}(t)=\frac{\left(\lambda_{1}-a_{1}\right)}{b_{1}} \cdot C_{1} \cdot e^{\lambda_{1} \cdot t}+\frac{\left(\lambda_{2}-a_{1}\right)}{b_{1}} \cdot C_{2} \cdot e^{\lambda_{2} \cdot t} .
\end{array}\right.
$$

Let's consider an example of using the model (1) to assess natural and technogenic risk in the case when the NTS is functionally similar to external natural and technogenic influences. A functionally similar external influence is understood as a type of external influence in which the effects of both natural and technogenic factors are described by the same functional dependencies.

The First case. We use the functional dependencies of the linear form:

$$
\left\{\begin{array}{l}
L_{1}^{(0)}(t)=\xi_{1}-\eta_{1} \cdot t \\
L_{2}^{(0)}(t)=\xi_{2}-\eta_{2} \cdot t
\end{array}\right.
$$

Find the general form of a partial solution for a nonuniform system of equations of the form (1). Let's represent the system (2) in the following form [12]:

while assuming: 


$$
\left\{\begin{array}{cc}
F_{11}(t)=e^{\lambda_{1} \cdot t} ; & F_{12}(t)=e^{\lambda_{2} \cdot t} ; \\
F_{21}(t)=\frac{\left(\lambda_{1}-a_{1}\right)}{b_{1}} \cdot e^{\lambda_{1} \cdot t} ; & F_{22}(t)=\frac{\left(\lambda_{2}-a_{1}\right)}{b_{1}} \cdot e^{\lambda_{2} \cdot t} .
\end{array}\right.
$$

We write (4) in matrix form:

$$
\left(\begin{array}{l}
r_{1}(t) \\
r_{2}(t)
\end{array}\right)=\left(\begin{array}{ll}
F_{11}(t) & F_{12}(t) \\
F_{21}(t) & F_{22}(t)
\end{array}\right) \times\left(\begin{array}{l}
C_{1} \\
C_{2}
\end{array}\right),
$$

or more compact: $R_{0}(t)=F(t) \cdot C_{0}$, где $R_{0}(t)$ - functions matrix of changing the components of natural and technological risk without considering external impact of the system (4); $F(t)$ - system matrix (5); $C_{0}$ - matrix of system coefficients (4).

In this case, the particular solution of system (1) corresponding to finding the matrix $\mathrm{R}(\mathrm{t})$ of changes functions in natural and technogenic risk due to the external influences on the NTS from the natural environment and the techno sphere, we will look for on the basis of the following relation $[13,14]$ :

$$
\tilde{R}(t)=F(t) \cdot \int F^{-1}(t) \cdot L(t) d t,
$$

where $F^{-1}(t)$ - the inverse matrix for $F(t) ; L(t)$ - the matrix of changes in the external natural and technogenic effects on the NTS.

Let's construct the matrix $F^{-1}(t)$, for this purpose we find the determinant of the matrix $\mathrm{F}(\mathrm{t})$ :

$$
\begin{gathered}
\Delta=\left|\begin{array}{ll}
F_{11}(t) & F_{12}(t) \\
F_{21}(t) & F_{22}(t)
\end{array}\right|=F_{11}(t) . \\
F_{22}(t)-F_{21}(t) \cdot F_{12}(t)=\frac{\left(\lambda_{2}-\lambda_{1}\right)}{b_{1}} . \\
e^{\left(\lambda_{1}+\lambda_{2}\right) \cdot t},
\end{gathered}
$$

The matrix of algebraic extensions $\bar{F}(t)$ for the matrix $\mathrm{F}(\mathrm{t})$ will have the following form:

$$
\bar{F}(t)=\left(\begin{array}{cc}
\frac{\left(\lambda_{2}-a_{1}\right)}{b_{1}} \cdot e^{\lambda_{2} \cdot t} & -\frac{\left(\lambda_{1}-a_{1}\right)}{b_{1}} \cdot e^{\lambda_{1} \cdot t} \\
-e^{\lambda_{2} \cdot t} & e^{\lambda_{1} \cdot t}
\end{array}\right),
$$

Then the matrix transposed with respect to the matrix (9) is written as:

$$
F^{T}(t)=\left(\begin{array}{cc}
\frac{\left(\lambda_{2}-a_{1}\right)}{b_{1}} \cdot e^{\lambda_{2} \cdot t} & -e^{\lambda_{2} \cdot t} \\
-\frac{\left(\lambda_{1}-a_{1}\right)}{b_{1}} e^{\lambda_{1} \cdot t} & e^{\lambda_{1} \cdot t}
\end{array}\right),
$$

Let us define the integrand functional matrix $\mathrm{U}(\mathrm{t})$ taking into account (10):

$$
U(t)=F^{-1}(t) \times L(t)=\frac{1}{\Delta} \times\left(\begin{array}{cc}
\frac{\left(\lambda_{2}-a_{1}\right)}{b_{1}} \cdot e^{\lambda_{2} \cdot t} & -e^{\lambda_{2} \cdot t} \\
-\frac{\left(\lambda_{1}-a_{1}\right)}{b_{1}} \cdot e^{\lambda_{1} \cdot t} & e^{\lambda_{1} \cdot t}
\end{array}\right)\left(\begin{array}{l}
L_{1}(t) \\
L_{2}(t)
\end{array}\right) .
$$

Consider a functionally similar effect on NTS of the form (3). These types of impacts describe the manifestations of movements that occur in the geological environment and leading to the occurrence of catastrophic seismic events observed in the territories of the district, local and "point" scale levels. They are characterized by sudden emergencies at techno sphere facilities, for example, explosions of equipment, collapse of buildings, structures, structures of various kinds.

In this case, the matrix of changes in the external natural and technological impacts on the NTS has the form:

Consider a functionally similar effect on NTS of the type (3). These types of influences describe the manifestations of movements that occur in the geological environment and lead to the occurrence of catastrophic seismic events observed on the territories of regional, local

$$
\left\{\begin{array}{l}
\chi_{1}(t)=\int u_{1}(t) d t=\frac{\left[-b_{1} \cdot \eta_{2}+\left(\lambda_{2}-a_{1}\right) \cdot \eta_{1}\right] \cdot e^{-\lambda_{1} \cdot t}}{\lambda_{1} \cdot\left(\lambda_{2}-\lambda_{1}\right)}+\tilde{C}_{1}, \\
\chi_{2}(t)=\int u_{2}(t) d t=\frac{\left[-\left(\lambda_{1}-a_{1}\right) \cdot \eta_{1}+b_{1} \cdot \eta_{2}\right] \cdot e^{-\lambda_{2} \cdot t}}{\lambda_{2} \cdot\left(\lambda_{2}-\lambda_{1}\right)}+\tilde{C}_{2},
\end{array}\right.
$$

where $\tilde{C}_{1}, \tilde{C}_{2}-$ constant coefficients, considering changes in natural and technogenic influences on the NTS at the initial time.

Considering (15), we will reveal the ratio (7): and "point" scale levels. They are characterized by sudden emergencies at techno sphere facilities, such as equipment explosions, collapses of buildings and structures of various types.

In this case, the matrix of changes in external natural and technogenic influences on the NTS has the form:

$$
L(t)=\left(\begin{array}{l}
\xi_{1}-\eta_{1} \cdot t \\
\xi_{2}-\eta_{2} \cdot t
\end{array}\right)^{\prime}=\left(\begin{array}{l}
-\eta_{1} \\
-\eta_{2}
\end{array}\right) .
$$

Define the components of the matrix $U(t)$ :

$$
u_{1}(t)=\frac{\left[\left(\lambda_{2}-a_{1}\right) \cdot L_{1}(t)-b_{1} \cdot L_{2}(t)\right] \cdot e^{-\lambda_{1} \cdot t}}{\lambda_{2}-\lambda_{1}} .
$$

By analogy, we obtain the expression for $u_{2}(t)$ :

$$
u_{2}(t)=\frac{\left[b_{1} \cdot L_{2}(t)-\left(\lambda_{1}-a_{1}\right) \cdot L_{1}(t)\right] \cdot e^{-\lambda_{2} \cdot t}}{\lambda_{2}-\lambda_{1}} .
$$

Integrating expressions (13) and (14):

$$
\tilde{R}(t)=F(t) \times \chi(t)=\left(\begin{array}{ll}
F_{11}(t) & F_{12}(t) \\
F_{21}(t) & F_{22}(t)
\end{array}\right) \times\left(\begin{array}{l}
\chi_{1}(t) \\
\chi_{2}(t)
\end{array}\right) .
$$

As a result, after the corresponding transformations, we get expressions for the functions of changes in natural and technogenic risk in the NTS: 


$$
\left\{\begin{array}{c}
\bar{r}_{1}(t)=D_{1} \cdot e^{\lambda_{1} \cdot t}+D_{2} \cdot e^{\lambda_{2} \cdot t}+\frac{1}{\lambda_{1} \cdot \lambda_{2}} \cdot \\
\quad\left[\left(\lambda_{1}+\lambda_{2}-a_{1}\right) \cdot \eta_{1}-b_{1} \cdot \eta_{2}\right] \\
\bar{r}_{2}(t)=\frac{\left(\lambda_{1}-a_{1}\right)}{b_{1}} \cdot D_{1} \cdot e^{\lambda_{1} \cdot t}+\frac{\left(\lambda_{2}-a_{1}\right)}{b_{1}} \cdot D_{2} \cdot e^{\lambda_{2} \cdot t}+ \\
+\frac{1}{b_{1} \cdot \lambda_{1} \cdot \lambda_{2}} \cdot\left[a_{1} \cdot b_{1} \cdot \eta_{2}+\left(\lambda_{1}-a_{1}\right) \cdot\left(\lambda_{2}-a_{1}\right) \cdot \eta_{1}\right]
\end{array}\right.
$$

where $D_{1}=C_{1}+\tilde{C}_{1} ; \quad D_{2}=C_{2}+\tilde{C}_{2}$.

The Second case. Let's now consider similar influences from natural and technogenic factors:

$$
\left\{\begin{array}{l}
L_{1}^{(0)}(t)=\xi_{1}-\eta_{1} \cdot t^{2} \\
L_{2}^{(0)}(t)=\xi_{2}-\eta_{2} \cdot t^{2}
\end{array}\right.
$$

Influences of this type (parabolic), for example, from the geological environment, characterize the effects of geodynamic hazards, the intensity of which gradually decreases with the distance from the epicenter of their manifestation. Such hazards include crepe, karstdeformation, subsidence, and landslide processes that

$$
\begin{aligned}
& \bar{r}_{1}(t)=\frac{2}{\left(\lambda_{1}-\lambda_{2}\right)}\left\{\begin{array}{l}
\frac{\left[\eta_{2} b_{1}-\eta_{1}\left(\lambda_{2}-a_{1}\right)\right]\left(1+\lambda_{1} t\right)}{\lambda_{1}^{2}}+ \\
+\frac{\left[\eta_{1}\left(\lambda_{1}-a_{1}\right)-\eta_{2} b_{1}\right]\left(1+\lambda_{2} t\right)}{\lambda_{2}^{2}}
\end{array}\right\}+D_{1} e^{\lambda_{1} \cdot t}+D_{2} e^{\lambda_{2} t}, \\
& \bar{r}_{2}(t)=\frac{2}{b_{1}\left(\lambda_{1}-\lambda_{2}\right)}\left\{\begin{array}{l}
\frac{\left(\lambda_{1}-a_{1}\right)\left[\eta_{2} b_{1}-\eta_{1}\left(\lambda_{2}-a_{1}\right)\right]\left(1+\lambda_{1} t\right)}{\lambda_{1}^{2}}+ \\
+\frac{\left(\lambda_{1}-a_{1}\right)\left[\eta_{1}\left(\lambda_{1}-a_{1}\right)-\eta_{2} b_{1}\right]\left(1+\lambda_{2} t\right)}{\lambda_{2}^{2}}
\end{array}\right\}+\frac{\left(\lambda_{1}-a_{1}\right)}{b_{1}} D_{1} e^{\lambda_{1} \cdot t}+\frac{\left(\lambda_{2}-a_{1}\right)}{b_{1}} D_{2} e^{\lambda_{2} \cdot t},
\end{aligned}
$$

where $D_{1}=C_{1}+\tilde{C}_{1} ; \quad D_{2}=C_{2}+\tilde{C}_{2}$.

\section{Risk modeling for functionally different natural and technogenic influences}

The Third case. Let technogenic influence reflect the manifestations of movements that occur in the geological environment, leading to the occurrence of catastrophic seismic events in the territories. These types of influences are reflected in the second equation (21). At the same time, natural influence are described by a parabolic equation (the first equation in (21)), the intensity of which gradually decreases with the distance from the epicenter of their manifestation, reflecting the so - called "slow" catastrophes-creep, karst-deformation, subsidence, landslide processes. cover the territories of local, district, and regional scale levels. For the techno sphere, examples include fires, chemical releases, and fallout of radioactive substances. Let us find a matrix of changes in the external natural and technogenic influences on NTS:

$$
L(t)=\left(\begin{array}{l}
\xi_{1}-\eta_{1} \cdot t^{2} \\
\xi_{2}-\eta_{2} \cdot t^{2}
\end{array}\right)^{\prime}=\left(\begin{array}{l}
-2 \eta_{1} \cdot t \\
-2 \eta_{2} \cdot t
\end{array}\right)
$$

We will search for a particular solution of system (1) for the case (18) by the method of variation of an arbitrary constant, finally obtaining:$$
\left\{\begin{array}{l}
L_{1}^{(0)}(t)=\xi_{1}-\eta_{1} \cdot t^{2} \\
L_{2}^{(0)}(t)=\xi_{2}-\eta_{2} \cdot t
\end{array}\right.
$$

where $\xi_{1}, \xi_{2}, \eta_{1}, \eta_{2}-$ some constant coefficients.

The matrix of changes in the external natural and technogenic influences on the NTS for (21) has the form:

$$
L(t)=\left(\begin{array}{c}
\xi_{1}-\eta_{1} t^{2} \\
\xi_{2}-\eta_{2} t
\end{array}\right)^{\prime}=\left(\begin{array}{c}
-2 \eta_{1} t \\
-\eta_{2}
\end{array}\right)
$$

or:

$$
\left\{\begin{array}{c}
L_{1}(t)=-2 \eta_{1} t \\
L_{2}(t)=-\eta_{2}
\end{array}\right.
$$

Applying the procedure described above, we define $C_{1}(t)$ and $C_{2}(t)$ as particular solutions of system (22) for the case of external natural and technogenic influences on NTS of the form (21):

$$
\begin{gathered}
C_{1}(t)=\frac{1}{\lambda_{1}^{2}\left(\lambda_{2}-\lambda_{1}\right)}\left[2 \eta_{1}\left(\lambda_{2}-a_{1}\right)\left(1+\lambda_{1} t\right)-b_{1} \eta_{2} \lambda_{1}\right] e^{-\lambda_{1} t}+\tilde{C}_{1}, \\
C_{2}(t)=\frac{\left[2 \eta_{1} \cdot\left(\lambda_{1}-a_{1}\right) \cdot\left(1+\lambda_{2} \cdot t\right)-b_{1} \cdot \eta_{2} \cdot \lambda_{2}\right] e^{-\lambda_{2} \cdot t}}{\lambda_{2}^{2} \cdot\left(\lambda_{1}-\lambda_{2}\right)}+\tilde{C}_{2},
\end{gathered}
$$

where $\tilde{C}_{1}, \tilde{C}_{2}-$ the constants of integration; $\lambda_{1}, \lambda_{2}-$ the roots of the uniform characteristic equation for (1).

Then the relations for the functions of changing the natural-technogenic risk for NTS in the case of joint functionally different influences of the species (21) from

$$
\left\{\begin{array}{c}
\bar{r}_{1}(t)=D_{1} \cdot e^{\lambda_{1} \cdot t}+D_{2} \cdot e^{\lambda_{2} \cdot t}+V_{1}^{(3)}(t), \\
\bar{r}_{2}(t)=\frac{\left(\lambda_{1}-a_{1}\right)}{b_{1}} \cdot D_{1} \cdot e^{\lambda_{1} \cdot t}+\frac{\left(\lambda_{2}-a_{1}\right)}{b_{1}} \cdot D_{2} \cdot e^{\lambda_{2} \cdot t}+V_{2}^{(3)}(t),
\end{array}\right.
$$


where $D_{l}, D_{2}-$ are constant coefficients that take into account changes in natural and technogenic influences on the NTS at the initial time, and the functions $V_{1}^{(3)}(t)$ and $V_{2}^{(3)}(t)$ are defined by equations:

$$
\left\{\begin{array}{c}
V_{1}^{(3)}(t)=\frac{b_{1} \eta_{2} \lambda_{1}-2 \eta_{1}\left(\lambda_{2}-a_{1}\right)\left(1+\lambda_{1} t\right)}{\lambda_{1}^{2}\left(\lambda_{1}-\lambda_{2}\right)}+\frac{2 \eta_{1}\left(\lambda_{1}-a_{1}\right)\left(1+\lambda_{2} t\right)-b_{1} \eta_{2} \lambda_{2}}{\lambda_{2}^{2}\left(\lambda_{1}-\lambda_{2}\right)}, \\
V_{2}^{(3)}(t)=\frac{\left(\lambda_{1}-a_{1}\right)\left[b_{1} \eta_{2} \lambda_{1}-2 \eta_{1}\left(\lambda_{2}-a_{1}\right)\left(1+\lambda_{1} t\right)\right]}{b_{1} \lambda_{1}^{2}\left(\lambda_{1}-\lambda_{2}\right)}+\frac{\left(\lambda_{2}-a_{1}\right)\left[2 \eta_{1}\left(\lambda_{1}-a_{1}\right)\left(1+\lambda_{2} t\right)-b_{1} \eta_{2} \lambda_{2}\right]}{b_{1} \lambda_{2}^{2}\left(\lambda_{1}-\lambda_{2}\right)} .
\end{array}\right.
$$

The Fourth case. We now consider the functionally differing effects of the kind (28).

$$
\left\{\begin{array}{l}
L_{1}^{(0)}(t)=\xi_{1}-\eta_{1} \cdot t^{2} \\
L_{2}^{(0)}(t)=\frac{1}{\xi_{2}+\eta_{2} \cdot t}
\end{array}\right.
$$

The natural influences is described by a parabolic equation, the first equation in (28), the intensity of which gradually decreases with distance from the epicenter of its manifestation, while the intensity of the technogenic

$$
C_{1}(t)=\frac{1}{\lambda_{1}-\lambda_{2}}\left\{2 \eta_{1}\left(\lambda_{2}-a_{1}\right) \int t e^{-\lambda_{1} t} d t-b_{1} \eta_{2} \int \frac{e^{-\lambda_{1} t}}{\left(\xi_{2}+\eta_{2} t\right)^{2}} d t\right\} .
$$

Analyzing (30), we see that taking the first integral isn't difficult, but the second one belongs to the class of "not taken".

For its approximate finding we decompose the integrand function

$$
f(t)=\frac{e^{-\lambda_{1} \cdot t}}{\left(\xi_{2}+\eta_{2} t\right)^{2}} .
$$

in a power series in a neighborhood of a point $t=t_{0}$.

$$
f^{\prime \prime}(t)=\frac{\left[\lambda_{1}^{2} \cdot\left(\xi_{2}+\eta_{2} \cdot t\right)^{2}+4 \lambda_{1} \eta_{2} \cdot\left(\xi_{2}+\eta_{2} \cdot t\right)+6 \eta_{2}^{2}\right) \cdot e^{-\lambda_{1} \cdot t}}{\left(\xi_{2}+\eta_{2} \cdot t\right)^{4}} .
$$

The $3^{\text {rd }}$ order derivative will be determined by the relation:

$$
f^{\prime \prime \prime}(t)=-\frac{\left[\lambda_{1}^{3} \cdot\left(\xi_{2}+\eta_{2} \cdot t\right)^{3}+6 \lambda_{1}^{2} \cdot \eta_{2} \cdot\left(\xi_{2}+\eta_{2} \cdot t\right)^{2}+18 \lambda_{1} \cdot \eta_{2}^{2} \cdot\left(\xi_{2}+\eta_{2} \cdot t\right)+24 \eta_{2}^{3}\right) \cdot e^{-\lambda_{1} t}}{\left(\xi_{2}+\eta_{2} \cdot t\right)^{5}} .
$$

Performing further differentiation of function (31), we arrive at a recurrence relation. Using it, we represent function (31) in a neighborhood of the point t0 in the form of a series expanded in powers of $\left(t-t_{0}\right)$ : Performing further differentiation of the function $(31)$, we come to a

$$
f(t)=f\left(t_{0}\right)+f^{\prime}\left(t_{0}\right) \cdot\left(t-t_{0}\right)+\frac{f^{\prime \prime}\left(t_{0}\right)}{2 !} \cdot\left(t-t_{0}\right)^{2}+\frac{f^{\prime \prime \prime}\left(t_{0}\right)}{3 !} \cdot\left(t-t_{0}\right)^{3}+\cdots+\frac{f^{(n)}\left(t_{0}\right)}{n !} \cdot\left(t-t_{0}\right)^{n}+\cdots
$$

The numerical estimates made by the authors showed that the necessary calculation accuracy of $0.001 \%$ is achieved by considering the first seven members of the series (35). Introducing the notation $W_{0}=f\left(t_{0}\right) ; W_{1}=$ $f^{\prime}\left(t_{0}\right) / 1$ !; $W_{2}=f^{\prime \prime}\left(t_{0}\right) / 2$ !; $\ldots ; \quad W_{6}=f^{(6)}\left(t_{0}\right) / 6$ !, we write function (31) in the following form:

$$
f(t)=\frac{e^{-\lambda_{1} \cdot t}}{\left(\xi_{2}+\eta_{2} \cdot t\right)^{2}}=\sum_{n=0}^{6} W_{n} \cdot \frac{\left(t-t_{0}\right)^{n}}{n !} .
$$

Then the second integral of expression (30) is represented as:

$$
\int \frac{e^{-\lambda_{1} \cdot t} d t}{\left(\xi_{2}+\eta_{2} \cdot t\right)^{2}}=W_{0} \cdot t+\sum_{p=1}^{6} \frac{W_{p} \cdot\left(t-t_{0}\right)^{p+1}}{(p+1) !}+C_{0}
$$


Finding the first integral from expression (30) and subsequently transforming this expression, we obtain the final relation for the function $C_{1}(\mathrm{t})$ :

$$
C_{1}(t)=\frac{1}{\lambda_{2}-\lambda_{1}}\left[\frac{2 \eta_{1}\left(\lambda_{2}-a_{1}\right)\left(1+\lambda_{1} t\right)}{\lambda_{1}^{2}} e^{-\lambda_{1} t}+b_{1} \eta_{2} X(t)\right]+\tilde{C}_{1},
$$

where $\tilde{C}_{1}-$ an arbitrary integration constant, and the function $X(t)$ is determined by the expression:

$$
X(t)=W_{0} \cdot t+\sum_{p=1}^{6} \frac{W_{p} \cdot\left(t-t_{0}\right)^{p+1}}{(p+1) !}
$$

Find the derivative of the function $C_{1}(t)$ :

$$
\begin{gathered}
C^{\prime}{ }_{1}(t)=\frac{1}{\lambda_{2}-\lambda_{1}}\left[2 \eta_{1}\left(\lambda_{2}-a_{1}\right) t e^{-\lambda_{1} t}-\right. \\
\left.b_{1} \eta_{2} \Phi(t)\right],
\end{gathered}
$$

where function $\Phi(t)$ is defined by the expression

$$
\Phi(t)=\sum_{q=0}^{6} W_{q} \cdot\left(t-t_{0}\right)^{q},
$$

and the coefficients $W_{q}$ have the same meaning as the expansion coefficients in (36).

Define, as shown above, the function $C_{2}(t)$.

$$
C_{2}(t)=\frac{2 \eta_{1}\left(\lambda_{1}-a_{1}\right)\left(1+\lambda_{2} t\right)}{\lambda_{1} \lambda_{2}\left(\lambda_{1}-\lambda_{2}\right)} e^{-\lambda_{2} t}+\frac{b_{1} \eta_{2}}{\left(\lambda_{1}-\lambda_{2}\right)^{2}}\left[\Phi(t)-\frac{1}{\lambda_{1}-\lambda_{2}} \Theta(t)\right] \cdot e^{\left(\lambda_{1}-\lambda_{2}\right) \cdot t}+\tilde{C}_{2},
$$

where functions $\Phi(t)$ and $\Theta(t)$ are defined by the expression:

$$
\begin{aligned}
& \left\{\begin{array}{l}
\Phi(t)=\sum_{q=0}^{6} W_{q} \cdot\left(t-t_{0}\right)^{q} ; \\
\Theta(t)=\sum_{\beta=0}^{5} G_{\beta} \cdot\left(t-t_{0}\right)^{\beta} .
\end{array}\right. \\
& \left\{\begin{array}{c}
\bar{r}_{1}(t)=D_{1} e^{\lambda_{1} t}+D_{2} e^{\lambda_{2} t}+\Omega_{1}(t) e^{\lambda_{1} t}+V_{1}^{(4)}(t), \\
\bar{r}_{2}(t)=\frac{\left(\lambda_{1}-a_{1}\right)}{b_{1}} D_{1} e^{\lambda_{1} t}+\frac{\left(\lambda_{2}-a_{1}\right)}{b_{1}} D_{2} e^{\lambda_{2} t}+\Omega_{2}(t) e^{\lambda_{1} t}+V_{2}^{(4)}(t),
\end{array}\right.
\end{aligned}
$$

Ultimately, the functions of changing the natural and techngenic risks in the NTS for the case of joint functionally different influences of the kind (28) from the environment and the techno sphere are presented in the

where $D_{1}, D_{2}-$ constant coefficients, functions $V_{1}^{(4)}(t)$ and

$V_{2}^{(4)}(t)$ are defined from equations:

$$
\left\{\begin{array}{c}
V_{1}^{(4)}(t)=\frac{2 \eta_{1}}{\lambda_{1}\left(\lambda_{1}-\lambda_{2}\right)}\left[\frac{\left(\lambda_{1}-a_{1}\right)\left(1+\lambda_{2} t\right)}{\lambda_{2}}-\frac{\left(\lambda_{2}-a_{1}\right)\left(1+\lambda_{1} t\right)}{\lambda_{1}}\right] \\
V_{2}^{(4)}(t)=\frac{2 \eta_{1}}{b_{1} \lambda_{1}\left(\lambda_{1}-\lambda_{2}\right)}\left[\frac{\left(\lambda_{2}-a_{1}\right)\left(\lambda_{1}-a_{1}\right)\left(1+\lambda_{2} t\right)}{\lambda_{2}}-\frac{\left(\lambda_{1}-a_{1}\right)\left(\lambda_{2}-a_{1}\right)\left(1+\lambda_{1} t\right)}{\lambda_{1}}\right],
\end{array}\right.
$$

and functions $\Omega_{1}(t)$ and $\Omega_{2}(t)$ - from equations:

$$
\left\{\begin{array}{c}
\Omega_{1}(t)=\frac{b_{1} \cdot \eta_{2}}{\lambda_{1}-\lambda_{2}}\left\{\frac{1}{\lambda_{1}-\lambda_{2}}\left[\Phi(t)-\frac{1}{\lambda_{1}-\lambda_{2}} \Theta(t)\right]-X(t)\right\}, \\
\Omega_{2}(t)=\frac{\eta_{2}}{\lambda_{1}-\lambda_{2}}\left\{\frac{\left(\lambda_{2}-a_{1}\right)}{\lambda_{1}-\lambda_{2}}\left[\Phi(t)-\frac{1}{\lambda_{1}-\lambda_{2}} \Theta(t)\right]-\left(\lambda_{1}-a_{1}\right) X(t)\right\} .
\end{array}\right.
$$

\section{Results}

1. In the article confirmed and implemented two new mathematical models of the dynamics of naturaltechnogenic risk arising in natural-technical systems under the influence of functionally similar impacts, as well as two new models of the dynamics of naturaltechnogenic risk arising in NTS under the influence of functionally different impacts.

2. In relation to this type of influences, the first model, which characterizes linear types of impacts, describes the manifestations of movements that occur in the geological environment and lead to the occurrence of catastrophic seismic events. These types of influences are characterized by sudden emergencies at techno sphere objects, such as equipment explosions, collapses of buildings and various structures. The second model of this type describes the effects of a parabolic type, the intensity of which gradually decreases with the distance from the epicenter of their manifestation. They describe crepe, karstdeformation, subsidence, landslide processes

3. The presented models of the second type differ from each other in the types of influences: the third model considers together parabolic (reflecting threats, the intensity of which gradually decreases with the distance from the epicenter) and linear types of influences (reflecting suddenly emerging threats), the fourth model - parabolic and hyperbolic (reflecting 
threats, the intensity of which decreases sharply over time) types of influences.

4. The general approach to modeling natural and technogenic risks, as well as the solutions presented, are aimed at using in analytical activities the services that carry out preventive work in connection with threats of natural and technogenic kind, responding to the consequences of realized threats, mainly the divisions of the EMERCOM of Russia that analyze the occurrence of risk situations and predict their development. The models considered are easily adaptable to account for external natural and manmade impacts of other types, such as exponential or oscillatory, which often occur in real life. In addition, the described theoretical approach to the construction of a dynamic model can be extended to other types of risks, for example, anthropogenic

5. A concrete example shows that in the case of complex types of influences on NTS described by functionally "difficult" mathematical relations, it is possible to apply their simplified representation in the form of expansion into series and be limited, depending on the required accuracy of calculations, to several initial terms of the series.

6. The experience of modeling has shown that mathematically it is possible to describe quite accurately almost any types of combined natural and man-made impact on natural and technical systems. Based on the results of this description, it is necessary to create a special "Atlas" of complex impacts on NTS in order to simulate various situations and develop the most effective response to emerging hazards.

\section{Acknowledgments}

This work was completed and published with the support of Russian Foundation for Basic Research, Project No. 19-07-00445.

\section{References:}

[1] Shoigu S. K., Vorobyov Yu. L., Vladimirov V. A. Catastrophes and the state. - Moscow: Energoatomizdat, 1997. - 160 p.

[2] Minaev V. A., Faddeev A. O. Safety and recreation: system view on the problem of risks / Proceedings of the II International scientific and practical conference "Tourism and recreation: fundamental and applied researches". April 20, 2007. - Moscow: Lomonosov Moscow State University, 2008. - Pp. 329-334.

[3] Minaev V. A., Faddeev A. O. Methods of assessment of geo-ecological risk and geo-ecological safety of landscape-territorial complexes / Proceedings of the seventeenth scientific and technical conference "Security Systems-2008". October 30, 2008. Moscow: Academy of the State Fire Service of the EMERCOM of Russia, 2008. - Pp. 96-102.

[4] Minaev V. A., Faddeev A. Geoecological risk Assessments. Modeling of tourist and recreational territories safety - M.: Finance and statistics, INFRAM, 2009. - 370 p.

[5] Abramova A.V., Bondar K. M., Danilov R. M., Minaev V. A.., Pavlova S. A., Popov A. N., Faddeev
A. O. Modeling of geodynamic risks in emergency situations: monograph / edited by K. M. Bondar, V. A. Minaev, A. O. Faddeev. - Khabarovsk: Far Eastern Law Institute of the Internal Affairs Ministry of Russia, 2014. - 124 p.

[6] Minaev V. A., Faddeev A. O., Kuzmenko N. A. Modeling and assessment of geodynamic risks. - M.: "RTSoft" - "Kosmoskop", 2017. - 256 p.

[7] Minaev V. A., Topolsky N. G., Faddeev A. O., Bondar K. M., Mokshantsev A.V. Geodynamic risks and construction. Mathematical models. - M.: Academy of the State Fire Service of the EMERCOM of Russia, 2017. - 208 p.

[8] Shoigu S. K., Vladimirov V. A., Vorobyov Yu. L. and others. Safety of Russia. Legal, socio-economic and scientific and technical aspects. Protection of the population and territories from emergency situations of natural and technogenic character. - M.: Znanie, 1999. - $162 \mathrm{p}$.

[9] Akimov V. A., Novikov V. D., Radaev N. N. Natural and technogenic emergencies: dangers, threats, risks. - M.: Delovoy Express, 2001. - 344 p.

[10]Burkov V. N., Graziansky E. V., Dzyubko S. I., Shchepkin A.V. Models and mechanisms of safety management. - M.: SINTEG Publishing house, 2001. $-160 \mathrm{p}$.

[11] Arnold V. I. Ordinary differential equations. - M.: MCSMA, 2012. - 344 p.

[12] Agafonov S. A., Muratova T. V. Ordinary differential equations. - M.: Academia, 2018. - 352 p.

[13]Kamke E. Handbook of ordinary differential equations. - Moscow: Nauka, 1971. - 576 p.

[14]Demidovich B. P., Modenov V. P. Differential equations. - SPb.: LAN, 2006. -288 p.

\section{About the authors}

Vladimir A. Minaev, Doctor of Engineering Sciences, Full Professor, Professor of Bauman Moscow State Technical University. E-mail: m1va@yandex.ru.

Rodion O. Stepanov, Ph.D. of Engineering Sciences, Associate Professor, Director of the Arctic Programs Directorate of Bauman Moscow State Technical University. E-mail: stepanovr@,bmstu.ru.

Alexander O. Faddeev, Doctor of Engineering Sciences, Associate Professor, Chief researcher of Bauman Moscow State Technical University. E-mail: faol@mail.ru 